\title{
ON A CONJECTURE CONCERNING THE MEANS OF THE EIGENVALUES OF RANDOM STURM-LIOUVILLE BOUNDARY VALUE PROBLEMS*
}

\author{
By WILliAM E. BOYCE (Rensselaer Polytechnic Institute)
}

\begin{abstract}
It has been known for several years that the expected value $\left\langle\lambda_{1}\right\rangle$ of the smallest eigenvalue of a self-adjoint positive definite random Sturm-Liouville boundary value problem satisfies the relation $\left\langle\lambda_{1}\right\rangle \leq \mu_{1}$, where $\mu_{1}$ is the smallest eigenvalue of the corresponding deterministic problem obtained by replacing each random coefficient by its mean. It has been an open question whether similar inequalities are valid for the higher eigenvalues. The answer is negative, as shown by the counterexample given in this note.
\end{abstract}

Consider the random Sturm-Liouville eigenvalue problem consisting of the differential equation

$$
L u=-\frac{d}{d x}\left[p(x, \omega) \frac{d u}{d x}\right]+q(x, \omega) u=\lambda u, \quad 0<x<1
$$

and the boundary conditions

$$
u(0)=u(1)=0
$$

The coefficients $p(x, \omega)$ and $q(x, \omega)$ are given stochastic processes, with $\omega \in \Omega$, where $(\Omega, \mathscr{F}, P)$ is the underlying probability space. We assume that, as functions of $x$ and with probability one, $p(x, \omega) \in C^{1}, q(x, \omega) \in C, p(x, \omega)>0$, and $q(x, \omega) \geq 0$ on $0 \leq x \leq 1$. In other words, these conditions hold except possibly on an $\omega$-set $N$ such that $P(N)=0$. For each $\omega$ in $\Omega \backslash N$ the problem (1), (2) has a sequence of eigenvalues

$$
0<\lambda_{1}(\omega)<\lambda_{2}(\omega)<\cdots<\lambda_{n}(\omega)<\cdots
$$

with the corresponding eigenfunctions

$$
u_{1}(x, \omega), u_{2}(x, \omega), \ldots, u_{n}(x, \omega), \ldots
$$

We assume that the eigenfunctions have been normalized so that

$$
\left(u_{i}, u_{j}\right)=\delta_{i j}
$$

where $\delta_{i j}$ is the Kronecker delta and $(u, v)=\int_{0}^{1} u(x) v(x) d x$. The explicit dependence of $\lambda_{i}$ and $u_{i}$ on $\omega$ emphasizes that each eigenvalue is a random variable and each eigenfunction a stochastic process.

Denoting the mathematical expectation by $\langle\cdot\rangle$, we can write

$$
\langle p(x, \omega)\rangle=p_{0}(x), \quad\langle q(x, \omega)\rangle=q_{0}(x),
$$

\footnotetext{
* Received October 9, 1979.
} 
and

$$
p(x, \omega)=p_{0}(x)+p_{1}(x, \omega), \quad q(x, \omega)=q_{0}(x)+q_{1}(x, \omega),
$$

where

$$
p_{0}(x)>0, \quad q_{0}(x) \geq 0
$$

and

$$
\left\langle p_{1}(x, \omega)\right\rangle=0, \quad\left\langle q_{1}(x, \omega)\right\rangle=0 .
$$

Associated with the problem (1), (2) is the nonrandom problem obtained by replacing $p(x, \omega)$ and $q(x, \omega)$ by their respective means $p_{0}(x)$ and $q_{0}(x)$ :

$$
\begin{gathered}
L_{0} w=-\frac{d}{d x}\left[p_{0}(x) \frac{d w}{d x}\right]+q_{0}(x) w=\mu w, \quad 0<x<1 \\
w(0)=w(1)=0 .
\end{gathered}
$$

The eigenvalues of the problem (7), (8) can also be ordered so that

$$
0<\mu_{1}<\mu_{2}<\cdots<\mu_{n}<\cdots
$$

and we will assume that the corresponding eigenfunctions $w_{1}(x), w_{2}(x), \ldots, w_{n}(x), \ldots$ have also been normalized so that

$$
\left(w_{i}, w_{j}\right)=\delta_{i j} .
$$

The principal purpose of this note is to clarify the relation between $\left\langle\lambda_{i}\right\rangle$ and $\mu_{i}$.

It is convenient to characterize the eigenvalues $\lambda_{i}$ and $\mu_{i}$ by means of minimum principles. First, we consider the smallest eigenvalues $\lambda_{1}$ and $\mu_{1}$. If

$$
D(u)=(L u, u)=\int_{0}^{1}\left(p u^{\prime 2}+q u^{2}\right) d x
$$

and if we define the class of admissible functions

$$
\mathscr{U}=\left\{u(x, \omega) \mid(u, u)=1, u(0)=u(1)=0, u \in C^{1} \text { for } \omega \in \Omega \mid N\right\}
$$

then for each $\omega \in \Omega \mid N$ it follows that

$$
\lambda_{1}(\omega)=\min _{\|} D(u)
$$

Similarly, we have

$$
\mu_{1}=\min _{w} D_{0}(w)
$$

where

$$
\begin{aligned}
D_{0}(w) & =\left(L_{0} w, w\right)=\int_{0}^{1}\left(p_{0} w^{\prime 2}+q_{0} w^{2}\right) d x, \\
\mathscr{W} & =\left\{w(x) \mid(w, w)=1, w(0)=w(1)=0, w \in C^{1}\right\} .
\end{aligned}
$$

Under the assumptions made here, it has been known for some time $[1,2]$ that

$$
\left\langle\lambda_{1}\right\rangle \leq \mu_{1} .
$$


This result is a consequence of the fact that the minimizing function $w_{1}(x)$ for the deterministic minimum principle (12) also belongs to $\mathscr{U}$ and hence is an admissible function for the random minimum principle (11). Thus, we have

$$
\lambda_{1} \leq \int_{0}^{1}\left(p w_{1}^{\prime 2}+q w_{1}^{2}\right) d x .
$$

By taking the expectation of both sides, we obtain

$$
\begin{aligned}
\left\langle\lambda_{1}\right\rangle & \leq \int_{0}^{1}\left(\langle p\rangle w_{1}^{\prime 2}+\langle q\rangle w^{2}\right) d x \\
& =\int_{0}^{1}\left(p_{0} w_{1}^{\prime 2}+q_{0} w^{2}\right) d x=D_{0}\left(w_{1}\right)=\mu_{1} .
\end{aligned}
$$

A practical consequence of (17) is that it affords a convenient estimate for $\left\langle\lambda_{1}\right\rangle$. Examples in [2] show that both the $<$ and $=$ in (17) can occur.

By using a result due to Courant [3], the argument used above to establish (17) can be extended to show that

$$
\left\langle\lambda_{1}\right\rangle+\cdots+\left\langle\lambda_{m}\right\rangle \leq \mu_{1}+\cdots+\mu_{m}
$$

for any positive integer $m$.

It is attractive to conjecture that

$$
\left\langle\lambda_{i}\right\rangle \leq \mu_{i}
$$

for $i=2,3, \ldots$ as well as for $i=1$. Unfortunately, in the minimum principles for the higher eigenvalues, the class of admissible functions is restricted by orthogonality conditions. For example, the minimum principle characterizing $\lambda_{2}$ has the class of admissible functions

$$
\mathscr{U}_{1}=\left\{u(x, \omega) \mid(u, u)=1, u(0)=u(1)=0, u \in C^{1},\left(u, u_{1}\right)=0 \text { for } \omega \in \Omega \mid N\right\},
$$

and similarly for $\mu_{2}$. Thus, there is no reason to believe that the minimizing function $w_{2}$ for the deterministic problem belongs to the admissible class $\mathscr{U}_{1}$ for the random problem, and the preceding argument fails. In fact, the conjecture (19) is false, as can be shown by the counterexample constructed below.

Let us now write (1) in the form

$$
L u=\left(L_{0}+\varepsilon L_{1}\right) u=\lambda u,
$$

where

$$
L_{0} u=-\left(p_{0} u^{\prime}\right)^{\prime}+q_{0} u, L_{1} u=-\left(p_{1} u^{\prime}\right)^{\prime}+q_{1} u,
$$

and $\varepsilon$ is a scaling parameter. Following Purkert and vom Scheidt [4], we seek to express $\lambda_{m}(\varepsilon, \omega)$ and $u_{m}(x, \varepsilon, \omega)$ in the form

$$
\begin{gathered}
\lambda_{m}(\varepsilon, \omega)=\sum_{j=0}^{\infty} k_{j}^{(m)}(\omega) \varepsilon^{j}, \\
u_{m}(x, \varepsilon, \omega)=w_{m}(x)+\sum_{\substack{j=1 \\
j \neq m}}^{\infty} c_{j}^{(m)}(\varepsilon, \omega) w_{j}(x),
\end{gathered}
$$


where

$$
c_{j}^{(m)}(\varepsilon, \omega)=\sum_{i=0}^{\infty} \gamma_{i j m}(\omega) \varepsilon^{i}
$$

These are regular perturbation expansions, and so converge for $|\varepsilon|$ sufficiently small.

Expressions for the coefficients in (22), (23), and (24) up to fourth order in $\varepsilon$ are given in [4]. Here it is sufficient to note that, up to second-order terms,

$$
\lambda_{m}=\mu_{m}+\left(L_{1} w_{m}, w_{m}\right) \varepsilon+\sum_{\substack{j=1 \\ j \neq m}}^{\infty} \frac{\left(L_{1} w_{m}, w_{j}\right)^{2}}{\mu_{m}-\mu_{j}} \varepsilon^{2}+\cdots .
$$

Since $\left\langle\left(L_{1} w_{m}, w_{m}\right)\right\rangle=0$ by $(6)$, it follows that

$$
\left\langle\lambda_{m}\right\rangle=\mu_{m}+\sum_{\substack{j=1 \\ j \neq m}}^{\infty} \frac{\left\langle\left(L_{1} w_{m}, w_{j}\right)^{2}\right\rangle}{\mu_{m}-\mu_{j}} \varepsilon^{2}+\cdots .
$$

One can also use (25) to obtain estimates of higher moments of $\lambda_{m}$; for example,

$$
\operatorname{var}\left(\lambda_{m}\right)=\left\langle\left(L_{1} w_{m}, w_{m}\right)^{2}\right\rangle \varepsilon^{2}+\cdots .
$$

Consider the example

$$
-u^{\prime \prime}+[1+\varepsilon \eta(x, \omega)] u=\lambda u, \quad u(0)=u(1)=0,
$$

where we assume that $\varepsilon$ and $\eta(x, \omega)$ are small enough that $|\varepsilon \eta| \leq 1$ with probability one for $0 \leq x \leq 1$. Here

$$
L_{0} u=-u^{\prime \prime}+u, \quad L_{1} u=\eta u, \quad\langle\eta\rangle=0 .
$$

The corresponding averaged problem is

$$
-w^{\prime \prime}+w=\mu w, \quad w(0)=w(1)=0,
$$

with the eigenvalues and normalized eigenfunctions

$$
\mu_{n}=1+n^{2} \pi^{2}, \quad w_{n}(x)=\sqrt{ } 2 \sin n \pi x .
$$

Then

$$
\begin{aligned}
\left(L_{1} w_{m}, w_{j}\right) & =2 \int_{0}^{1} \eta(x, \omega) \sin m \pi x \sin j \pi x d x \\
& =\int_{0}^{1} \eta(x, \omega)[\cos (m-j) \pi x-\cos (m+j) \pi x] d x .
\end{aligned}
$$

We now choose

$$
\eta(x, \omega)=A(\omega) \cos k \pi x, \quad 0<k<m
$$

so that the random perturbation is a cosine wave with a random amplitude. Then by a simple calculation that exploits the orthogonality of the cosines, it follows that

$$
\begin{aligned}
\left(L_{1} w_{m}, w_{j}\right) & =A(\omega) / 2, \text { if } j=m-k, m+k \\
& =0, \text { otherwise. }
\end{aligned}
$$


Thus, the $\varepsilon^{2}$ term in (26) becomes

$$
\begin{aligned}
& \sum_{\substack{j=1 \\
j \neq m}}^{\infty} \frac{\left\langle\left(L_{1} w_{m}, w_{j}\right)^{2}\right\rangle}{\mu_{m}-\mu_{j}} \varepsilon^{2}=\underset{4 \pi^{2}}{\left\langle A^{2}\right\rangle}\left(\frac{1}{m^{2}-(m-k)^{2}}+\frac{1}{m^{2}-(m+k)^{2}}\right) \\
& =\frac{\left\langle A^{2}\right\rangle}{2 \pi^{2}\left(4 m^{2}-k^{2}\right)}>0 .
\end{aligned}
$$

Hence

$$
\left\langle\lambda_{m}\right\rangle=1+m^{2} \pi^{2}+\frac{\left\langle A^{2}\right\rangle}{2 \pi^{2}\left(4 m^{2}-k^{2}\right)} \varepsilon^{2}+O\left(\varepsilon^{3}\right) .
$$

Consequently,

$$
\left\langle\lambda_{m}\right\rangle>\mu_{m}
$$

provided only that $\varepsilon$ is small enough so that the higher-order terms in (31) can be neglected. By choosing $k=1$, it follows that (32) is true simultaneously for all $m \geq 2$. Thus, the conjecture (19) is definitely false in general.

A similar analysis can be applied to other types of self-adjoint positive definite problems, such as matrix eigenvalue problems or boundary-value problems having more general boundary conditions or higher-order differential equations.

\section{REFERENCES}

[1] W. E. Boyce, Random vibrations of elastic strings and bars, in Proc. 4th U.S. Nat. Cong. Appl. Mech. (1962), $77-85$

[2] W. E. Boyce, Random eigenvalue problems, in Probabilistic methods in applied mathematics, vol. 1, A. T. Bharucha-Reid (editor), Academic Press, New York, 1968, 1-73

[3] R. Courant and D. Hilbert, Methods of mathematical physics, vol. 1, Interscience, 1953, p. 459

[4] W. Purkert and J. vom Scheidt, Zur approximativen Lösung des Mittelungsproblems für die Eigenwerte stochastischer Differentialoperatoren, ZAMM, 57, 515-525 (1977) 